\title{
SURGICAL PROBLEMS OF WAR
}

This contribution is the third of four articles written by a Canadian surgeon who had considerable experience of war surgery in 1914-18

\section{HEAD WOUNDS : AMPUTATIONS}

BY

\section{AMBROSE L. LOCKW OOD, D.S.O., M.C.}

\section{Head Wounds}

The whole problem of gunshot wounds of the head was simplified when the necessity for and possibility of early operation were recognized. It was especially important to deal with such wounds before deep-seated infection in the substance of the brain was established and while it was possible to carry out total excision of the scalp wound and immediate suture. The important points in surgery of gunshot wounds of the head are:

1. The earliest possible evacuation to the casualty clearing station.

2. Accurate location of the missile, if retained; and determination of the extent of the fracture and position of bone fragments that may have been carried in. In addition, to ascertain whether there is a fracture of the base, with bleeding from the ear, or of the frontal plate, with escape of cerebrospinal fluid from the nose, and whether the lateral and longitudinal sinuses are involved.

3. A select anaesthesia, preferably local, and sufficient pre-operative sedation or intravenous hypnosis to allay nervous irritability and excitement.

4. Total excision of the wound of the scalp down to the bone.

5. Thorough cleansing of the exposed bone with a nonirritating antiseptic, and excision of enough bone to expose a quarter of an inch of healthy dura about the track of the missile through the dura and no more. This is particularly important if the dreadful hernia cerebri that occurred in nearly all gunshot wounds of the head operated upon at certain base hospitals is to be avoided.

6. Excision of the frayed devitalized track of the missile through the dura, but no more.

7. Careful irrigation with saline, and suction of the tract to clear out all foreign matter, disorganized and destroyed brain tissue, and blood clot; and, only then, careful exploration for the retained missile or fragment of bone. A catheter is best for this purpose, but occasionally a finger can be safely passed along the track.

8. If there is no penetration of the dura, but if there is an area that is dark, lustrous, boggy, non-pulsatile, and with disorganized brain matter and blood clot underlying it, do not hesitate to make a short stellate incision and carefully tease out all clot and broken-down brain matter, irrigate gently with saline, and, if possible, lightly resuture the dura, otherwise leave it open. Opening of the dura in such wounds is important. I have had patients who during operation under local anaesthesia became conscious almost at once. In addition the prolonged headache and stupor that persisted when the dura was not opened were almost entirely avoided, as was the danger of retained blood clot with the development of an organized haematoma that often undergoes cystic degeneration, with all the grave sequelae that brain surgeons have come to recognize after civilian injuries to the skull and that too often are overlooked. Rigid asepsis must, however, be maintained.

9. After removal of the missile and all other foreign matter, primary suture of the scalp wound-without drainage if dealt with early enough, otherwise leaving a small Penrose drain in a dependent position down just to the dura for forty-eight hours. Any wound of the scalp can be closed by a reversed " $S$ " (2) incision; all other methods to ensure closure of the scalp, such as lateral incisions, undue freeing of the scalp, bevelling incisions, etc., should be abandoned. It is important to realize the value of such a closure.

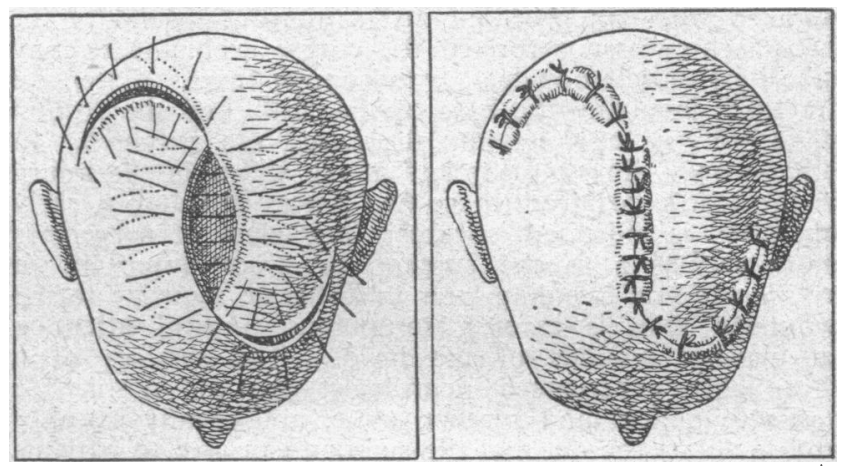

FIG. 1.-Reversed " $S$ " incision to ensure sliding flaps that may be best employed to close any gaping wound of the scalp.
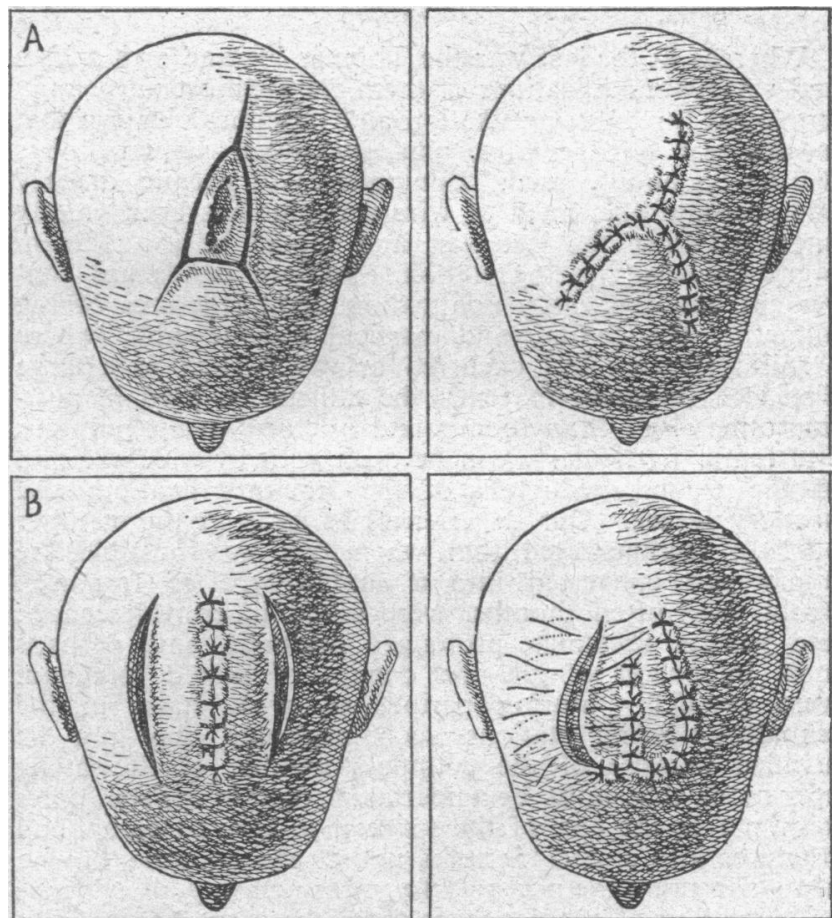

FIG. 2.-A. "Isle of Man" incision for wide " $\mathrm{V}$ " wound B. Undercutting incisions to permit closure of wounds: both are bad.

10. If there is a rent with loss of tissue in the longitudinal sinus, the lateral sinuses, or even in the torcular Herophili, use a postage-stamp patch of the thinnest of epicranial tissue or of fascia lata if available, but do not pack with gauze or plug with muscle. Cut the thinnest possible patch of epicranial tissue or of fascia lata, preferably the former, and merely appose it over the hole in the sinus. Hold it gently with a finger for one minute. It adheres and is held by cohesion, the circulation is re-established, and after two or three minutes such a patch can only with difficulty be removed. We have reorganized almost an entire torcular Herophili 
by such a method, requiring two or three such patches. It is important to maintain the circulation in the sinuses. Periodic lumbar punctures with withdrawal of varying quantities of spinal fluid, depending on the pressure of the fluid, may be necessary for a few days following operation, and particularly after late operation with infection and extensive loss of bone, when there is a greater tendency to hernia cerebri.

11. Head cases should be retained in the unit after operation for some days until the patient is entirely conscious and until all possible early complications have been avoided or have subsided.

12. It must be particularly kept in mind that the surgeon who can proclaim that a gunshot wound of the head is hopeless or foretell the course such a case may take has himself not dealt with enough cases. Extensive fractures involving the base of the skull, and the frontal plate with an opening into the nares, are excepted. In other words, almost regardless of the extent of the injury to the head apart from those two classes of fracture, early operation is indicated. Scarcely any class of surgery is more satisfying to the surgeon or more important in saving life and avoiding later unfortunate sequelae to the wounded than is careful, scrupulous surgical attention in gunshot wounds of the head. The services of a thoroughly experienced neurologist are of inestimable value to patient and surgeon alike, and greatly simplify and facilitate the aid that the surgeon can give to patients with wounds of the head. Such an internist should be attached to every casualty clearing station.

\section{Fractures}

Whereas in the last war the Thomas leg and arm splints and various modifications of them were almost universally employed for ensuring fixation and traction, I believe that in this war flexible wire, pins, and other similar things will be commonly used. Equipment that permits of easy and accurate placement of wire and pins has been greatly improved since the last war, and should be available in every casualty clearing station. Parham bands are convenient and are easily adapted in open fractures of the humerus, the femur, and particularly the tibia where a spiral fracture has occurred or where there is a large displaced fragment that may be difficult to hold in position otherwise. Many compound fractures of the humerus and femur particularly can be dealt with by wide excision of the wound, reduction of the fracture, fixation, and primary suture. Our experiences as early as the summer of 1916 demonstrated that when this was possible the injury was converted into a simple type of fracture; weeks, and often months, were saved in convalescence; and painful dressings, prolonged drainage, and, perhaps most important of all, the danger of mixed infection, were avoided. During periods of great stress in the casualty clearing stations such measures may not be justified because of the countless number of life-saving operations that must be undertaken, but in quieter times it should be the aim of the surgeon to convert compound fractures into closed fractures if that can be done, having always in mind the necessity for clearing away all evidence or possibility of gas gangrene from the wound or wounds. The use of plates, metal, or bone should be discouraged.

\section{Amputations}

Probably next in importance to the saving of human life in the surgery of a casualty clearing station is the accurate determination whether amputation is necessary or if the limb or limbs can be saved. Unfortunately, huge numbers of woanded constantly pass through a busy casualty clearing station for whom immediate amputation is life-saving and their only chance. Care must be taken, however, to be always certain that nothing less is possible. Amputation as a rule is necessary when:

1. There is massive gas gangrene of the limb that can no longer be removed by ablation of muscles.
2. There is destruction of the main artery and nerves.

3. There is a double compound fracture of the same bone of the limb with much soiling and destruction of tissue.

4. There is such extensive compound fracturing and displacement of component bones of a joint that reduction and fixation is impossible and even resection would not ensure a useful limb.

5. There is, in late cases that have lain out for many days, a purulent infection of a joint or muscle planes such that even after free drainage has been established the patient's life is endangered from absorption.

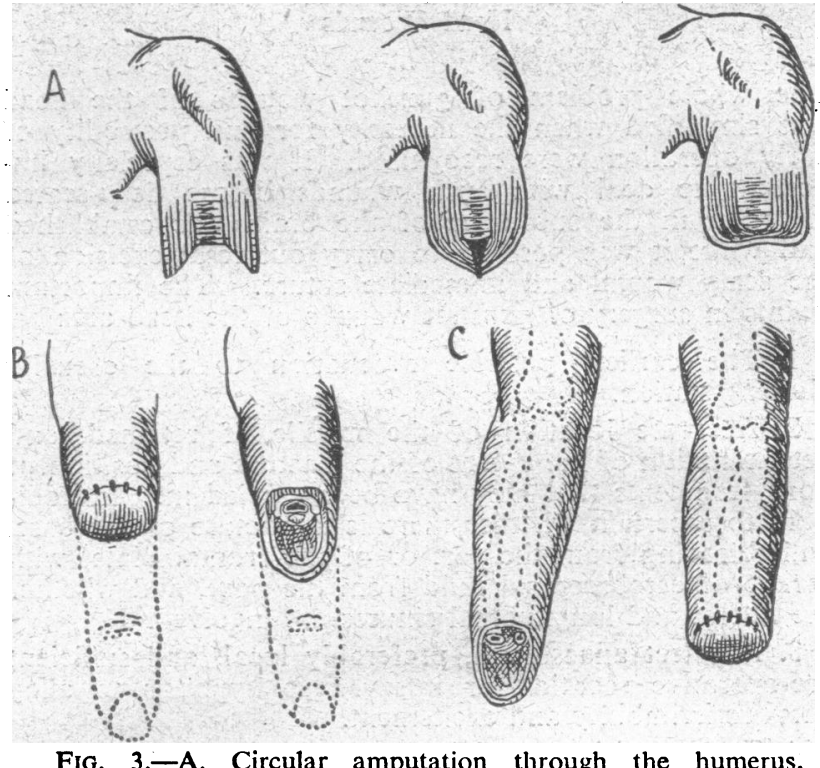

FIG. 3.-A. Circular amputation through the humerus. B. Flap amputation through
through the lower arm.

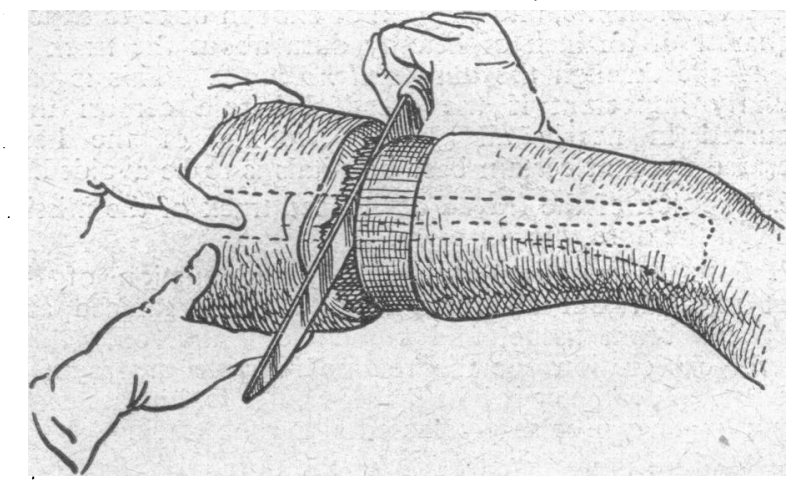

Fig. 4.-Circular flap amputation through the thigh.

Having decided to amputate, every care must be taken (1) to choose the best site ; (2) to plan and fashion flaps accurately so as to avoid pressure on the scar later, tension after suture, or indeed a redundant stump ; (3) to ligate main arteries securely and to shorten the nerves; (4) to section the bone accurately, not removing too much or too little, or splintering the periphery of the bone; (5) to maintain complete asepsis so far as it is possible ; (6) to give close attention to the wound for the ensuing days till danger of sepsis is past. I would draw attention to the value of circular amputation, not flush through the skin, muscle, and bone, but with a cuff of skin, and slightly higher section of the muscles and then of bone so that further surgical intervention is unnecessary other than perhaps skin suture to ensure a good useful limb.

Circular amputation, flush, the so-called guillotine amputation, is rarely warranted for any reason. It leaves an extensive raw surface to dress and necessitates a secondary procedure to close it. A modified circular 
amputation as suggested above takes only a few minutes longer to carry out, and in the great majority of cases can be sutured at once or later, and even if left open with traction on the skin will granulate in and heal itself. In addition, it permits as good drainage if lightly packed with gauze.

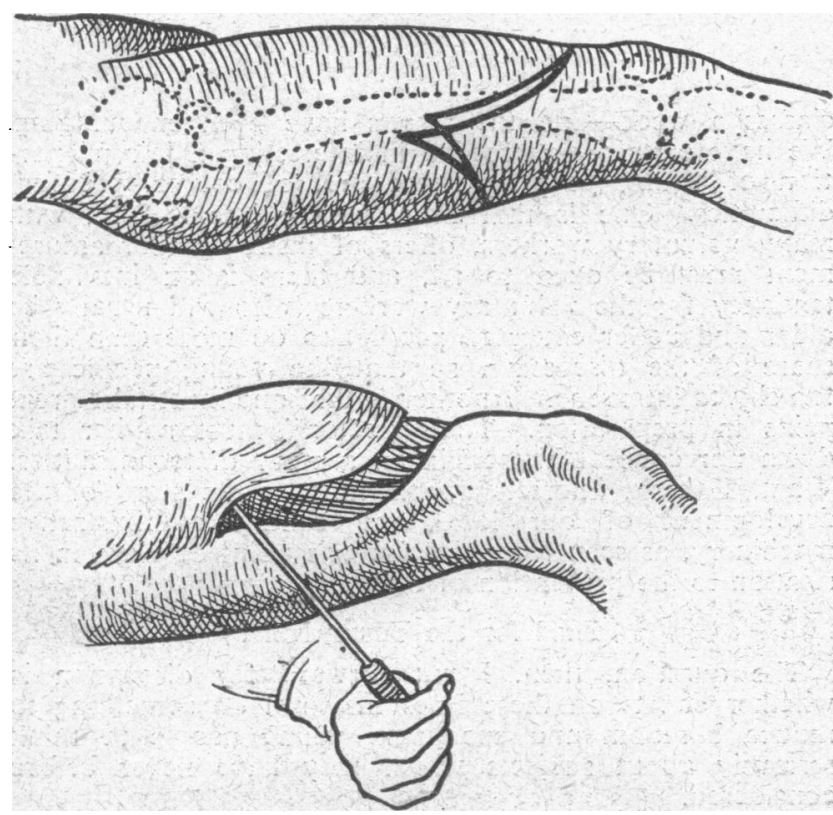

Fig. 5.-Flap amputation through the thigh.
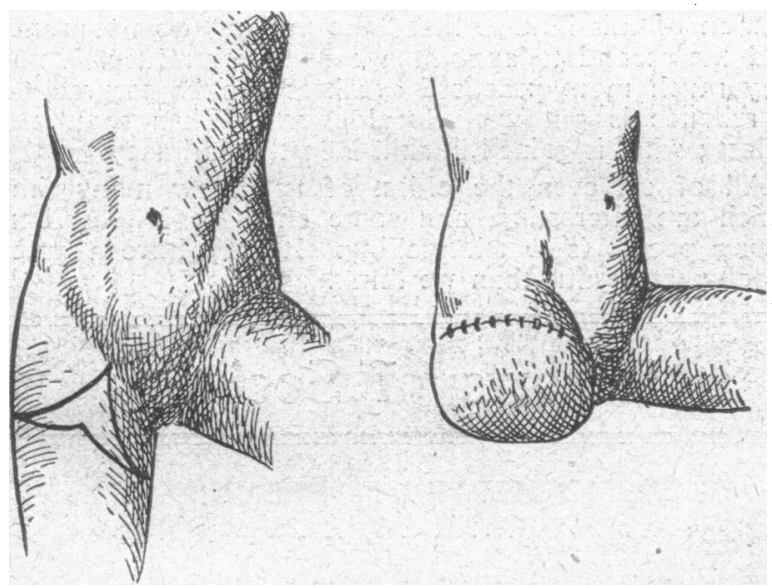

Fig. 6.-Y-flap amputation through the hip.

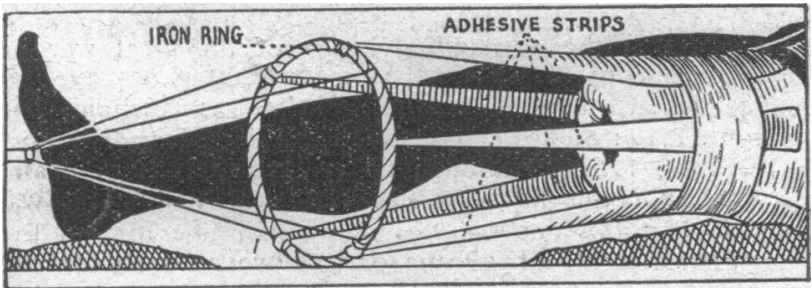

FIG. 7.-Method for preventing or overcoming retraction in circular amputations.

Odier, early in the nineteenth century, first described enlargements or swellings which develop on the distal end of a proximal segment of a peripheral nerve after partial or complete division. Nerves should be gently freed, injected with absolute alcohol for $2 \mathrm{~cm}$., and sectioned just below the injected area. They should not be crushed or ligated. The perineural flap method as suggested by Corner, the swing door or reversed $V$ operation, and crush-and-tie procedure have all been shown by the careful studies of Huber and Lewis not to prevent neuroma formation. In addition, one should not tie a nerve filament in with an artery: it is a direct cause of painful convalescence. It is a wise precaution to leave main arteries long and quickly insert a circular suture

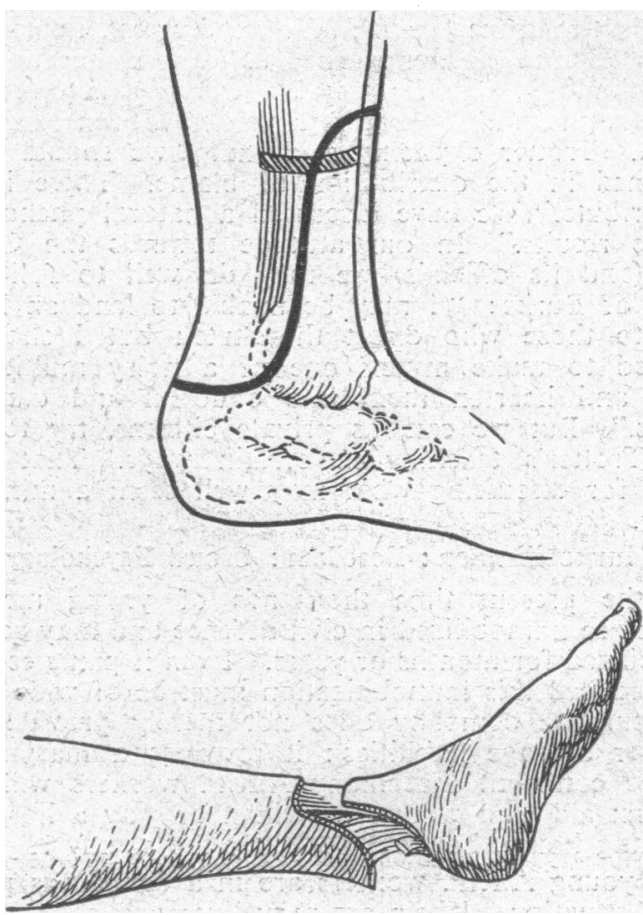

Fig. 8.-Incision for posterior flap amputation.

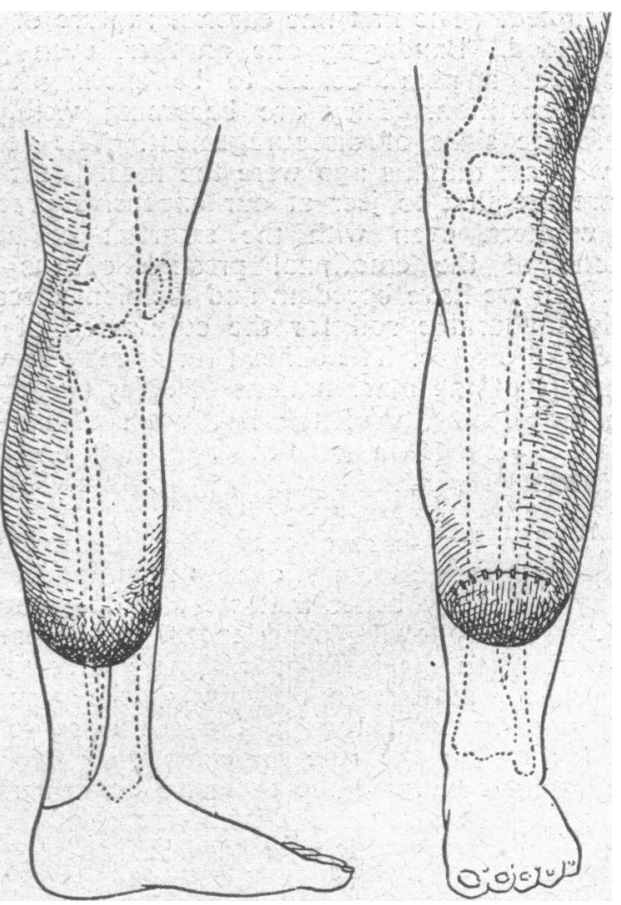

Fig. 9.-Result of posterior flap operation.

in the adjacent muscle and approximate it over the artery so as to avoid secondary haemorrhage.

While amputations should be elementary in principle, the fact is too often overlooked in practice that every amputation should be planned as a geometrical, mechanical, and anatomic problem. All young surgeons dealing with traumatic surgery should have at hand Farabeuf's great volume, which is ritualistic in its principles of amputation. 